\title{
Establishment of the hybrid endoscopic full-thickness resection of gastric gastrointestinal stromal tumors
}

\author{
HIROHITO MORI, HIDEKI KOBARA, SHINTARO FUJIHARA, NORIKO NISHIYAMA, MAKI AYAGI, \\ TAE MATSUNAGA, TATSUO YACHIDA and TSUTOMU MASAKI
}

Department of Gastroenterology and Neurology, Kagawa University, Miki, Kita, Kagawa 761-0793, Japan

Received January 13, 2014; Accepted September 3, 2014

DOI: $10.3892 / \mathrm{mco} .2014 .412$

\begin{abstract}
A prospective observational study was conducted to establish the procedure of hybrid endoscopic full-thickness resection (EFTR) using an existing flexible endoscope. The present study included 16 patients who underwent hybrid EFTR between September 2009 and February 2013 for gastric gastrointestinal stromal tumor (GIST). The patients were selected using the following inclusion criteria for histological findings: Mitotic counts $<5 /$ high-power field and immunohistochemical stains positive for KIT or cluster of differentiation 34 (CD34). The mean patient age was 68.2 years (range, 44-81 years); the male-to-female ratio was 6:10; lesion sites at upper (U), middle (M) and lower regions (L) of the stomach were $9 / 6 / 1$; and the average tumor diameter was $28.3 \mathrm{~mm}$. The mean surgical time was $271 \mathrm{~min}$ and the surgical time became progressively faster with each successive surgery. There were 12 and four patients with mitotic counts of $<5$ and 5-10, respectively, which was significantly different $(\mathrm{P}=0.01)$. Immunohistochemical stains showed that tumors from 13 and 10 patients (81.2 and 62.5\%, respectively) were positive for KIT and $\mathrm{CD} 34$, respectively $(\mathrm{P}=0.328)$. All resected surgical margins were negative. According to Fletcher's risk classification, there were five, eight and three patients at an 'extremely low', 'low', and 'intermediate' risk (31.2, 50 and $18.8 \%$, respectively) $(\mathrm{P}=0.003)$. The mean postoperative hospital duration was 12.3 days (range, 10-15 days). In conclusion, an ultra-minimally invasive surgery-hybrid EFTR is a safe and established surgical endoscopy procedure.
\end{abstract}

Correspondence to: Professor Tsutomu Masaki, Department of Gastroenterology and Neurology, Kagawa University, 1750-1 Ikenobe, Miki, Kita, Kagawa 761-0793, Japan

E-mail: hiro4884@med.kagawa-u.ac.jp

Key words: gastrointestinal stromal tumor, hybrid endoscopic full-thickness resection, ultra-minimally invasive surgery, mean surgical time, mean hospital stay

\section{Introduction}

Hybrid endoscopic full-thickness resection (EFTR) for the treatment of gastric gastrointestinal stromal tumors (GISTs) has become an endoscopic and laparoscopic surgery cross-technique. An increasing number of studies have focused on hybrid EFTR, which is performed in combination with endoscopy and laparoscopy (1-3). The indications for hybrid EFTR include GISTs that do not require lymphadenectomy and can be cured by radical tumor enucleation. Although a number of animal experiments using hybrid natural orifice translumenal endoscopic surgery (NOTES) have been reported, studies conducted on humans are required (4-6).

\section{Patients and methods}

Patients and identification of GISTs. The present study included 16 patients who underwent hybrid EFTR between September 2009 and February 2013 for the treatment of gastric GIST. The study was approved by the Ethics Committee of Kagawa University (Kagawa, Japan), and enrollment was conducted using the University Hospital Medical Information Network (no. 000004722 and 000008691). Written informed consent was obtained from each patient.

The GIST risk factors were evaluated using the Fletcher classification (?) as follows: Tumor size; histological findings, including mitosis; and immunohistochemical staining. The tumor size was measured with an endoscopic ultrasound (EUS) (20-MHz radial scan). Patients with a tumor size $<40 \mathrm{~mm}$ were selected for the study as GISTs that are $>40 \mathrm{~mm}$ cannot be removed through the esophagus. However, in the present study, four patients were included with tumors $>40 \mathrm{~mm}(42,54,46$ and $51 \mathrm{~mm})$. An EUS-fine-needle aspiration (FNA) was performed, as well as an en bloc biopsy using endoscopic submucosal dissection (ESD) for histopathology, as described previously (7). For small GISTs, $\sim 10 \mathrm{~mm}$, an en bloc biopsy was performed using ESD techniques as EUS-FNA is difficult to perform with small targets. Patients were selected using the following inclusion criteria for histological findings: Mitotic counts $<5 /$ high-power field (HPF) and immunohistochemical stains positive for KIT or cluster of differentiation 34 (CD34). The patients with tumors $>50 \mathrm{~mm}$, mitotic counts $>10 / 50 \mathrm{HPF}$ or immunohistochemical stains negative for KIT or CD34 were excluded from the study due 

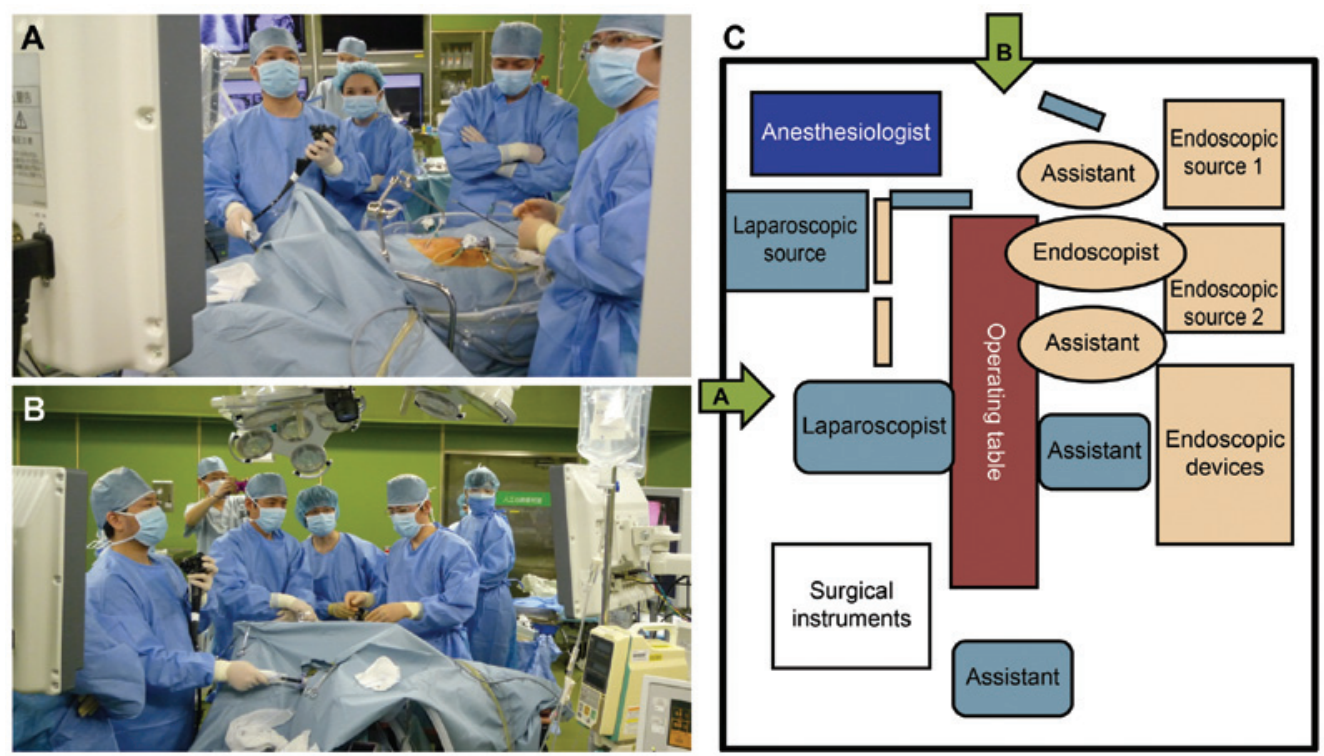

Figure 1. Configuration and schematic diagram of the operating room. (A) Configuration as observed from the right side of the patient. Two sources of light for the flexible endoscope and a stand for the endoscopic device were placed on the left side of the patient. The flexible endoscope operator and the assistant were also positioned on the left side of the patient. (B) Configuration as observed from the side of the patient's head.
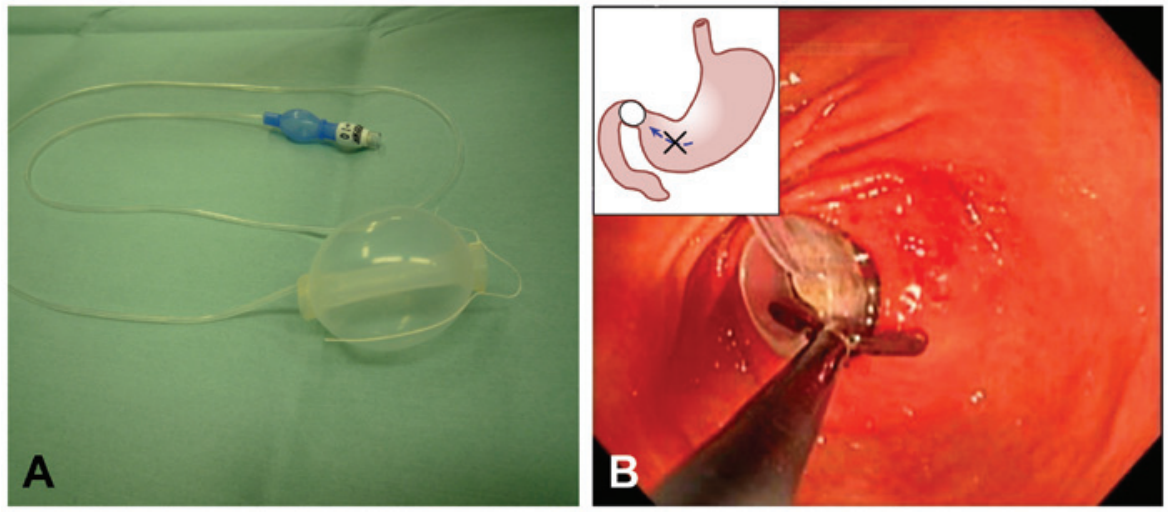

Figure 2. Duodenal balloon occlusion method. (A) A nylon thread was used to make a loop at the tip of a balloon that was designed for use in esophageal variceal sclerotherapy (length, $52 \mathrm{~mm}$; inner diameter, $10 \mathrm{~mm}$ ). (B) The loop was grasped with forceps, inserted into the duodenal bulb, inflated with $60-70 \mathrm{ml}$ of air and placed in an indwelling manner.

to the high-associated risk for GISTs. The study examined the EFTR success rate, surgical time (duration of the surgical procedure), presence or absence of complications, and length of hospital stay.

Statistical analysis. Data are presented as the mean \pm standard deviation values. Patient baseline statistics were analyzed using the Kruskal Wallis H-test, unpaired t-test and Mann-Whitney U test. The mitosis count and positive number of KIT/CD34 were analyzed using the unpaired t-test. The histological risk was analyzed using the Kruskal Wallis H-test and Mann-Whitney $\mathrm{U}$ test. $\mathrm{P}<0.05$ was considered to indicate a statistically significant difference. The statistical analysis was performed using Graph Pad Prism version 5 for Windows (GraphPad Software, San Diego, CA, USA).

Arrangement of the operating room. Two sources of light for the flexible endoscope and a stand for the endoscopic device were placed on the left side of the patient. The laparoscope operator was positioned in a manner similar to that of the conventional arrangement in laparoscopic surgery (Fig. 1A and B).

Operative devices. The following operative devices were used: i) Endoscopes: GIF type Q260J, GIF type H260Z and GIF type XP260NS (sterilized with EtO gas; Olympus Co., Tokyo, Japan); ii) incisional knives: Dual knife (KD-650L; Olympus Co.) and insulation-tipped (IT)-knife-2 (KD-611L; Olympus Co.); iii) hemostatic forceps: Coagrasper (FD-410LR; Olympus Co.); iv) tip attachment: Elastic Touch Attachment (TOP Co., Tokyo, Japan); v) incisional device: ERBE VIO300D (ERBE Elektromedizin GmbH, Tübingen, Germany); and vi) $\mathrm{CO}_{2}$ insufflation device: UCR (Olympus Co.); vii) Endo Catch (Covidien Japan, Tokyo, Japan) .

Surgical procedure. The surgery was performed under general anesthesia with the supervision of an anesthesiologist. A longitudinal 20-mm incision was made in the umbilical region, and the following laparoscopic ports were created: A $12-\mathrm{mm}$ 

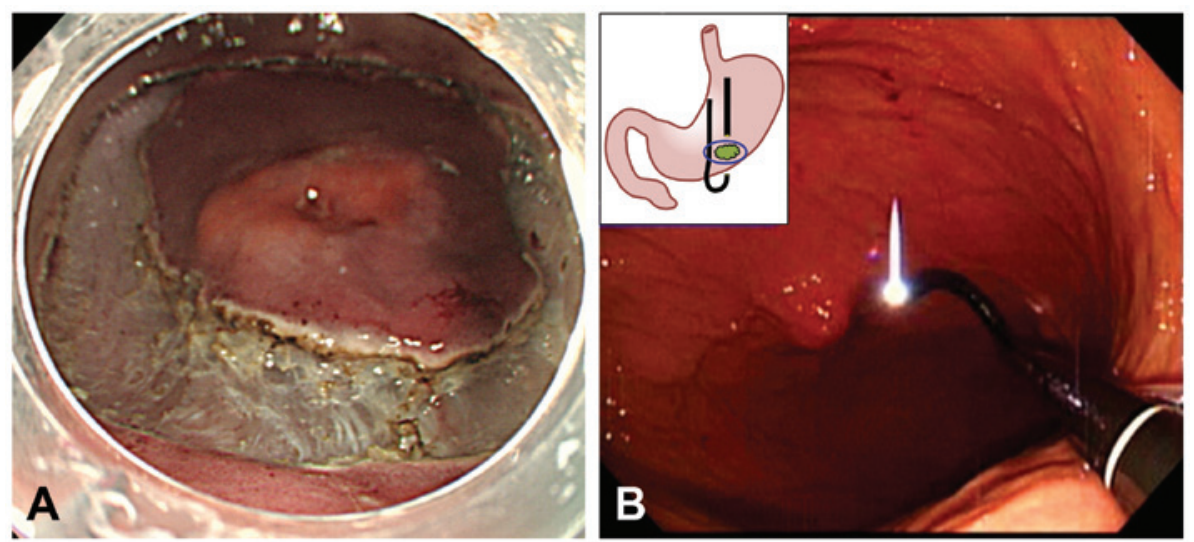

Figure 3. Endoscopic submucosal dissection (ESD) and double-scope methods. (A) Use of the ESD method in tumors that are surrounded by only muscle and serosa allows for a 10-mm incision to be opened that was as deep as the submucosal layer. (B) A perforation hole giving passage to a 5-mm diameter transnasal endoscope was opened in the incision; the endoscope was inserted into the abdominal cavity from the perforation hole and inverted intraperitoneally. A full-thickness resection of the gastric wall was subsequently performed safely, while a transnasal endoscope was used for observation.
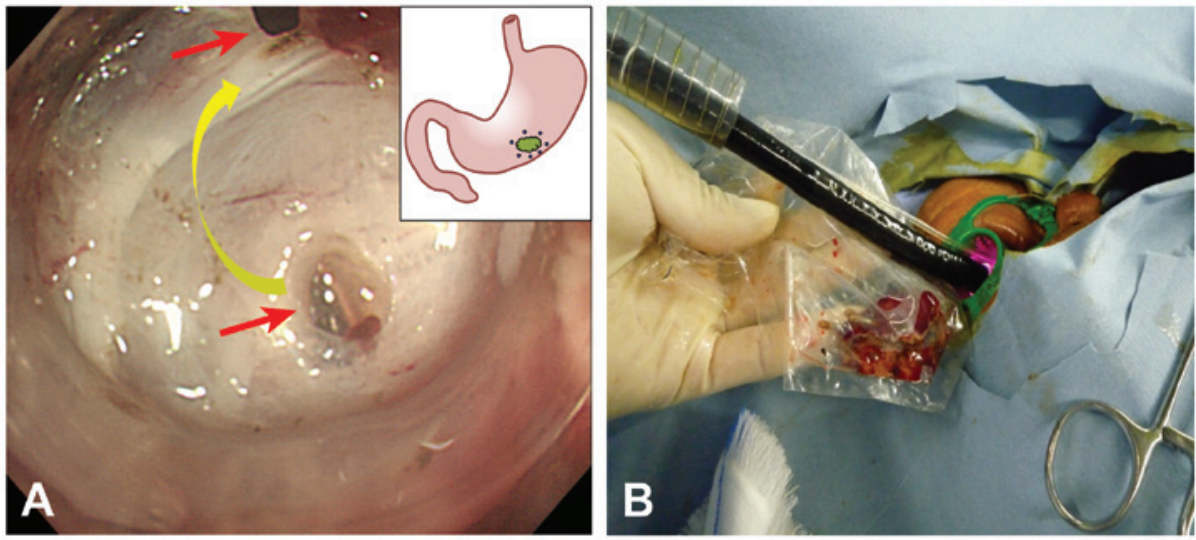

Figure 4. Equal perforation method. (A) In the incision of the muscle layer/serosa, perforation holes were placed at 5-mm intervals and the orifices of the holes were marked to secure a reliable incision line at the time of stomach collapse. A full-thickness resection was performed by connecting two small holes. (B) The tumor was collected through the mouth using an Endo Catch.

camera port, two $12-\mathrm{mm}$ ports at the upper right and left umbilical regions and a 5-mm port in the left upper quadrant of the abdomen. Using a nylon thread, a loop was created at the tip of a balloon designed for use in esophageal variceal sclerotherapy (length, $52 \mathrm{~mm}$; inner diameter, $10 \mathrm{~mm}$ ) (TOP Co.) (Fig. 2A). The balloon was inserted into the duodenal bulb and inflated with 60-70 $\mathrm{ml}$ of air (using the duodenal balloon occlusion method) (Fig. 2B) (8). The esophagus and stomach were carefully lavaged with $2,000 \mathrm{ml}$ of normal saline to disinfect the area in preparation for the full-thickness resection of the gastric wall (9). With an 8-mm safety margin around the tumor, preparations were made for a full incision into the submucosal layer and for an incision into the muscle layer (the incisions were made in accordance with the ESD method) (Fig. 3A) (4). The incision was widened to accommodate the 5-mm diameter transnasal endoscope. The transnasal endoscope (GIF N260) was inserted into the abdominal cavity, and the full-thickness resection was performed with the transoral endoscope (GIF Q260J), while the procedure was observed through the transnasal endoscope (the double-scope technique) (Fig. 3B) (4). The ERBE VIO300D was used as the incisional generator device. Perforation holes were placed at 5-mm intervals to secure a reliable incision line at the time of stomach collapse. The full-thickness resection was performed using the IT-knife- 2 and by connecting two small holes, the openings of which were of equal distance from the gastric wall (Fig. 4A) (4). Following the tumor excision, a full-thickness suture was placed under laparoscopy. The tumor was collected through the mouth using an Endo Catch (Covidien Japan) (Fig. 4B).

\section{Results}

Patient characteristics. The patient characteristics were as follows: Mean age of 68.2 years (range, 44-81 years); male-to-female ratio of $6: 10$, with no significant difference $(\mathrm{P}=0.627)$; lesion sites at upper $(\mathrm{U})$, middle $(\mathrm{M})$ and lower (L) regions of the stomach $(\mathrm{U} / \mathrm{M} / \mathrm{L})$ of $9 / 6 / 1$, with a significant difference between tumor locations. Almost all GISTs were located in the $\mathrm{U}$ to $\mathrm{M}$ regions $(\mathrm{P}=0.005)$; and the average tumor diameter was $28.3 \mathrm{~mm}$ (range, $8-54 \mathrm{~mm}$ ) (Table I). A total of nine hybrid EFTR procedures were performed in the U region, including three cases in which the proximal margins were within $1.5 \mathrm{~cm}$ of the esophagogastric junction. In the latter 
Table I. Patient characteristics.

\begin{tabular}{lcc}
\hline Variables & Patients $(\mathrm{n}=16)$ & P-value \\
\hline Age, mean (range), year & $68.2(44-81)$ & $0.627 \mathrm{NS}^{\mathrm{a}}$ \\
Gender (male/female) & $6 / 10$ & $0.005^{\mathrm{c}}\left(\mathrm{H}=10.5^{\mathrm{b}}\right)$ \\
Tumor location (U/M/L) & $9 / 6 / 1(56.3 \% / 37.5 \% / 6.2 \%)$ & \\
Tumor size mean (range), mm & $28.3(8-54)$ &
\end{tabular}

${ }^{\mathrm{a}}$ Unpaired t-test; ${ }^{\mathrm{b}} \mathrm{Kruskal}$ Wallis H-test; ${ }^{\mathrm{C}}$ Mann-Whitney U test. U; upper region, M; middle region, L; lower region of stomach.

Table II. Results of patients.

\begin{tabular}{lcc}
\hline Variables & Patients $(\mathrm{n}=16)$ & P-value \\
\hline EFTR completion/shift to laparotomy & $16 / 0$ & $271(100-480)$ \\
EFTR operation time, mean (range), min & $12 / 4(75 \% / 25 \%)$ & $0.01^{\mathrm{a}}$ \\
Mitosis (/50 high power field) $<5 / 5-10$ & $13 / 10(81.2 \% / 62.5 \%)$ & $0.328 \mathrm{NS}^{\mathrm{a}}$ \\
Immunohistochemical stain KIT/CD34 & $5 / 8 / 3 / 0(31.2 \% / 50 \% / 18.8 \%)$ & ${ }^{c} 0.003\left({ }^{\mathrm{b}} \mathrm{H}=12.36\right)$ \\
Histological risk & $12.3(10-15)$ & \\
Extremely low/low/intermediate/high (Fletcher classification) & & \\
Hospitalization, mean (range), days & &
\end{tabular}

${ }^{a}$ Unpaired t-test; ' Kruskal Wallis H-test; ' ${ }^{2}$ ann-Whitney U test. EFTR, endoscopic full-thickness resection; CD34, cluster of differentiation 34. NS, not significant.

cases, an 8-mm safety margin was created from the proximal side of the tumor margin. In all three cases, the surgical margins were negative. EFTR was successfully performed in all the patients and there were no complications requiring conversion to open surgery, even in cases with tumors $>40 \mathrm{~mm}$. The mean surgical time was $271 \mathrm{~min}$ (range, 100-480 min), and the surgical time became progressively faster with each successive surgery. In the first seven cases, the surgical times were 360-480 $\mathrm{min}$, whereas the surgical times for the last nine cases were 100-150 min. The surgical time appeared to be longer when the tumors were located in the $U$ region of the stomach. As the sample size was small, a correlation between the tumor location and surgical time could not definitively be established.

Results obtained. The pathological safety margin from the cut end to the tumor was an average of $8 \mathrm{~mm}$ (range, 6-11 $\mathrm{mm}$ ). All resected tumors were within the resected masses. There were 12 and four patients with mitotic counts of $<5$ and $5-10$, respectively, with a significant difference $(\mathrm{P}=0.01)$. Immunohistochemical stains showed that tumors from 13 and 10 patients (81.2 and 62.5\%) were positive for KIT and CD34, respectively $(\mathrm{P}=0.328)$. The resected surgical margins were negative. According to Fletcher's risk classification, there were $5 / 8 / 3$ patients at 'extremely low', 'low', and 'intermediate' risk $(31.2 / 50 / 18.8 \%)$, respectively $(\mathrm{P}=0.003)$. High-risk patients were not included. The mean postoperative hospital stay was 12.3 days (range, 10-15 days) (Table II). Patient safety was of primary importance, and therefore, the mean hospital stay was longer than the hospital stays for laparoscopic surgery. There were no complications, including bleeding, peritonitis, anastomotic leakage or stenosis.

\section{Discussion}

Several techniques have been suggested for the local resection of GIST, mainly laparoscopic local gastric resection $(10,11)$, laparoscopic and endoscopic cooperative surgery (LECS) (12), intragastric surgery (13) and hybrid EFTR (NOTES) (14). Of these procedures, hybrid EFTR has the advantage of enabling the use of electric scalpels (including the IT-knife-2 and dual knife). These scalpels are designed for flexible endoscopes and enable detailed and minimal tumor resection, even when a curved incision line or complex incision line along the tumor contour is made. As the sample size was small, the surgical time was shortened to approximately the same length as that of laparoscopic local gastric resection, indicating that surgical time is not an issue in hybrid EFTR. However, this experimental procedure has certain limitations. One limitation is that there have not been any randomized trials to compare hybrid EFTR with conventional techniques. Multi-center trials prior to the clinical acceptance of hybrid EFTR should therefore be conducted. The surgical procedure was designed to ensure incisional accuracy and excisional safety using the following steps: Setting the incision line according to the ESD method, using the double-scope technique to make and visualize the incision, creating the holes at equal distances from the abdominal wall (4) and using the duodenal balloon occlusion method (8). If hybrid EFTR is performed in accordance with 
these surgical procedures, LECS and hybrid EFTR may be established as less invasive, tailored surgical options.

In conclusion, hybrid EFTR, which is an ultra-minimally invasive surgery, is a safe and established surgical endoscopy procedure.

\section{Acknowledgements}

The authors acknowledge the Kagawa NOTES project for supporting the present study.

\section{References}

1. Kaouk JH, Haber GP, Goel RK, et al: Pure natural orifice translumenal endoscopic surgery (NOTES) transvaginal nephrectomy. Eur Urol 57: 723-726, 2010.

2. Isariyawongse JP, McGee MF, Rosen MJ, Cherullo EE and Ponsky LE: Pure natural orifice transluminal endoscopic surgery (NOTES) nephrectomy using standard laparoscopic instruments in the porcine model. J Endourol 22: 1087-1091, 2008.

3. Isayama H, Kogure H and Koike K: Endoscopic transgastric pure NOTES cholecystectomy with naso-gallbladder drainage tube placement and injection of a hyaluronic acid mixture (with Video). J Hepatobiliary Pancreat Sci 18: 106-111, 2011.

4. Mori H, Kobara H, Kobayashi M, et al: Establishment of pure NOTES procedure using a conventional flexible endoscope: review of six cases of gastric gastrointestinal stromal tumors. Endoscopy 43: 631-634, 2011.

5. Martínek J, Ryska O, Filípková T, et al: Natural orifice transluminal endoscopic surgery vs laparoscopic ovariectomy: complications and inflammatory response. World J Gastroenterol 18: 3558-3564, 2012.
6. Vieira JP, Linhares MM, Caetano EM Jr, et al: Evaluation of the clinical and inflammatory responses in exclusively NOTES transvaginal cholecystectomy versus laparoscopic routes: an experimental study in swine. Surg Endosc 26: 3232-3244, 2012.

7. Kobara H, Mori H, Fujiwara S, Nishiyama N, Kobayashi M and Masaki T: Bloc biopsy by tunneling method using the endoscopic submucosal dissection for upper gastrointestinal submucosal tumor. Endoscopy 44 (Suppl 2): E197-E198, 2012.

8. Mori $\mathrm{H}$, Kobara $\mathrm{H}$, Inoue $\mathrm{H}$, et al: New technique for safer endoscopic submucosal dissection using the duodenal balloon occlusion method. J Gastroenterol Hepatol 27: 81-85, 2012.

9. Mori H, Kobara H, Rafiq K, et al: Effects of gastric irrigation on bacterial counts before endoscopic submucosal dissection: a randomized case control prospective study. PLoS One 8: e65377, 2013.

10. Choi SM, Kim MC, Jung GJ, et al: Laparoscopic wedge resection for gastric GIST: long-term follow-up results. Eur J Surg Oncol 33: 444-447, 2007

11. Warsi AA and Peyser PM: Laparoscopic resection of gastric GIST and benign gastric tumours: evolution of a new technique. Surg Endosc 24: 72-78, 2010.

12. Hiki N, Yamamoto Y, Fukunaga T, et al: Laparoscopic and endoscopic cooperative surgery for gastrointestinal stromal tumor dissection. Surg Endosc 22: 1729-1735, 2008.

13. Hara J, Nakajima K, Takahashi T, et al: Laparoscopic intragastric surgery revisited: its role for submucosal tumors adjacent to the esophagogastric junction. Surg Laparosc Endosc Percutan Tech 22: 251-254, 2012.

14. Abe N, Takeuchi H, Ooki A, et al: Recent developments in gastric endoscopic submucosal dissection: towards the era of endoscopic resection of layers deeper than the submucosa. Dig Endosc 25 (Suppl 1): 64-70, 2013 